\title{
Zwischen Versorgungsengpässen und Forschungshemmnissen
}

\author{
Drei besonderen Herausforderungen muss sich die deutsche \\ Hämatologie und Onkologie stellen: Der Kluft zwischen mehr Krebs- \\ patienten und weniger Onkologen, die Verfügbarkeit von Zytostatika \\ sicherzustellen und die Rahmenbedingungen für klinische Forschung \\ zu verbessern. Die DGHO hat die Herausforderungen angenommen.
}

U nter dem Titel „Future Demands in Oncology - wie viel Forschung braucht die Versorgung?" resümierte der geschäftsführende Direktor der Deutschen Gesellschaft für Hämatologie und Onkologie (DGHO), Gerhard Ehninger, Dresden, im Rahmen einer Pressekonferenz auf der Jahrestagung der Gesellschaft in Stuttgart diese drei Punkte als diejenigen, die sich als wesentlich für die zukünftige Versorgung herausgestellt haben. Sein Rezept gegen die nachlassende Attraktivität des Berufs des Onkologen ist Nachwuchsarbeit. So bot der DGHO-Kongress in diesem Jahr für Studenten einen speziellen Orientierungstag, um dem potenziellen Nachwuchs den Beruf vorzustellen. Dazu wurden Nachwuchspreise verliehen und Ehninger kündigte einen Ausbau des wissenschaftlichen europäischen Austausches an, u. a. durch Harmonisierung der Weiterbildungsinhalte. Die deutsche und die österreichische Fachgesellschaft gingen daher eine Kooperation mit der European Society for Medical Oncology (ESMO) ein. Die DGHO-Weiterbildungskommission überarbeitet nun die deutsche Weiterbildungsordnung auf Grundlage internationaler Curricula, die österreichische Fachgesellschaft ÖGHO hat diesen Prozess bereits beendet.

\section{Versorgungsverantwortung bei Herstellern}

Versorgungsengpässe bei Zytostatika erfordern laut Ehninger entsprechende gesetzgeberische Maßnahmen. „Ursprünglich war im Arzneimittelgesetz in der 16. Novelle vorgesehen, dass ein PharmaLieferant, der die Zulassung hat, auch lie- fern muss oder bestraft wird“, erläuterte Ehninger. „Das wurde durch Lobbyismus bei der Verabschiedung gekippt und nun kann jeder, der eine Zulassung hat, liefern oder nicht liefern." Der Effekt, den er beobachtet: „Es wird, just in time“ geliefert, es gibt kaum mehr Lagerhaltung und lebenswichtige Medikamente, deren Patent bereits verfallen ist, werden sehr lieblos behandelt." 2012 habe es sechs Substanzen gegeben, die entweder bereits seit 12 Monaten nicht lieferbar waren oder immer wieder nicht verfügbar seien - wichtige Medikamente für lebensbedrohliche Erkrankungen. Ehninger stellte deshalb eine Ad-hoc-Arbeitsgruppe in Aussicht, die auch das Bundesministerium für Gesundheit (BMG) mit einbeziehen soll. Ein Ziel ist, die Hersteller stärker in die Versorgungspflicht und -verantwortung zu nehmen. Die Fachgesellschaft habe dazu bereits eine Liste erstellt, so Ehninger weiter.

\section{Globalisierter Handel gefährdet Versorgung}

Ein besonderer Dorn im Auge ist ihm das Verhalten auf dem Arzneimittelmarkt. „Es geht da wie auf einem ,Spotmarkt' zu“, sagte Ehninger und erläuterte, was er damit meint: Großhändler entschieden bei guten Preisen beispielsweise in England, die Medikamente palettenweise dorthin zu liefern und zu verkaufen, sodass diese dann in Deutschland nicht mehr lieferbar seien. So etwas sei auch im BMG bekannt. Langfristig könne auch das reiche Deutschland bei diesen Marktbedingungen „hinten runter fallen“. „Wir wissen auch von den ,Big Five-Pharmafirmen der Welt, dass in China vierfache Preise gezahlt werden, nur um dort eine Lieferung für die zunehmende Versorgung zu ermöglichen. Dabei werden dort derzeit nur $10 \%$ der Bevölkerung versorgt. Wahrscheinlich werden wir irgendwann Bittsteller werden, um überhaupt noch etwas abzubekommen", so Ehninger.

\section{Einheitliche ethische Basis für Europa}

Auch bezüglich der Hindernisse, die europaweiten klinischen Studien im Wege stehen, fand Ehninger klare Worte: Jedes Land habe seine eigenen Zulassungsbedingungen, seine eigenen Rahmenbedingungen, seine eigenen Ethikkommissionen. Bei Studien zur akuten myeloischen Leukämie müsse er alleine in Deutschland 45 Ethikkommissionen berücksichtigen, berichtete Ehninger. Wenn man eine europäische Studie mache, habe man gar 130 Ethikkommissionen und 27 Oberbehörden mit unterschiedlichen Unterlagen zu bedienen - Container voller Papier.

Deshalb forderte er: „Wir brauchen eine neue EU-Direktive, weil unter den derzeitigen Bedingungen in Europa keine multinationalen Studien möglich sind.“ Es müsse beispielsweise bei Studien, die nicht risikobehaftet sind (z. B. mit Ultraschalluntersuchungen), ein Ethikvotum für ganz Europa ausreichen. Ehninger beschwor den gemeinsamen europäischen Kulturkreis mit seinen grundsätzlich ähnlichen Vorstellungen von, gut' und ,richtig ' und trat damit Kritikern entgegen, die eine „mittelalterliche Forschungsethik" befürchteten [Sahm S, FAZ 24.09.2012]. Ehninger bekräftigte: Grundlagen, die nach den Menschenversuchen im dritten Reich in der Deklaration von Helsinki gelegt worden sind, werden natürlich weiterhin erfüllt. Aber die zeitliche Verzögerung von zehn bis 15 Jahren von der ersten klinischen Studie bis zur Zulassung könne mit weniger Bürokratie und weniger Überregulation verringert werden - zum Wohle des Patienten.

Friederike Klein 